\title{
A Case Report
}

\section{Acrylamide Versus Agarose Efficiency to recover Trace DNA (without PCR) on 70\% Ethylated Swab Versus Distilled Water Swab from Spectacles}

\author{
Simon Martin Manyanza Nzilibili ${ }^{\text {a,c }}$, M Sc. Forensic Science Student \\ Muh. Abduh Dwi Putraa , M Sc. Forensic Science Student \\ Ahmad Yudianto ${ }^{\text {abd }}$, Lecturer \\ a Forensic Science Program, Post Graduate School, Airlangga University, Surabaya - Indonesia. \\ b Department of Forensic and Medico-legal, Faculty of Medicine, Airlangga University, Surabaya - Indonesia c \\ Ministry of Health, Community Development, Gender Elderly and Children, Dodoma - Tanzania. \\ d Human Genetic Laboratory, Institute of Tropical Disease, Airlangga University, Surabaya - Indonesia.
}

\section{ABSTRACT :}

Introduction : Swabbing method is one of the established methods in recovering forensic traces. The used swab is suggested to be effective while in a moist state. Different fluids have been proposed then with minimal highlight to ethylated swabbing (prepared from ethanol) which is also found usable and friendly during extraction and storage of sample among other efficacy. Material and Methods : This study analyzes the significant use of $70 \%$ ethylated swab run simultaneously with distilled water swab to potentiate the findings under $6 \%$ acrylamide and $2 \%$ agarose electrophoresis without PCR. Besides assessing the use of these swabs (70\% ethyl and distilled water) and its electrophoretic reaction on the two gel, this study also studied the usefulness on spectacle as a potential source of traces.

Results : 70\% ethylated swab is found to concentrate DNA sample $(1421 \mu \mathrm{g} / \mathrm{ml})$ two times more compared to distilled water swab $(654.5 \mu \mathrm{g} / \mathrm{ml})$. In terms of purity of recovered DNA, $70 \%$ ethylated swab presented nearly similar measurement to distilled water swab (1.219 to 1.176 purity ratio respectively). Agarose electrophoresis was not able to detect DNA molecule hence the absence of visualized DNA fragments as band contrast from both swabs. On acrylamide gel electrophoresis, DNA molecule migration displayed multiple bands contrast with $70 \%$ ethylated swab; deep and concentrated towards positive electrode while lighter bands lagged near the negative end. Distilled water swab contrasted one deep band near the negative terminal electrode.

Conclusion : $6 \%$ acrylamide gel electrophoresis in absence of PCR is effective to profile trace DNA compared to agarose. In addition, 70\% ethylated swab is a useful method in recovering trace DNA to successful DNA analysis. Also, the spectacle is a potential evidence for trace DNA either found at the crime scene for criminal linkage or purposed forensic investigation.

\section{Corresponding Author \\ Simon Martin Manyanza Nzilibili}

Forensic Science Program, Postgraduate School, Airlangga

University, 4-6 Airlangga Rd., 60286 Surabaya, Indonesia

Contact : +255765434320

E-mail : nzilibili@gmail.com

\section{Article History:}

Received: 31 October 2018

Received in revised form: 30 November 2018

Accepted on: 30 November 2018

Available online: 1 June 2019

KEYWORDS : 6\% Acrylamide, 2\% Agarose, PC, 70\% Ethyl Swab, DNA, Spectacle , Trace evidence

\section{INTRODUCTION :}

Recovery of micro to macro Deoxyribonucleic acid (DNA) trace evidence is studied to involve adhesive tape, forceps and vacuum methods ${ }^{[1,2]}$. Swab method besides is also discussed and presented to work properly and far better while moist in recovering such trace evidence $\left.\mathrm{l}^{2-7}\right]$. Opted and used moisturizing fluid, in that case, remains of high and efficacy determinant. To explore the effectiveness, experimental studies done have explored the success of ethyl moist swabbing though most still recommend water moistening option ${ }^{[3,8]}$. Thus, in the DNA retrieval study; $95 \%$ ethanol swab was found suitable to recover DNA at high yield than distilled water similar to ${ }^{[10]}$, in which a $25 \%$ ethylated alcohol swab yielded the most compared to $50 \%$ and distilled water swab. Emphasis is on maximized retention of minute natured sample residing on swab after recovery against loss or blowaway ${ }^{[1]}$ Contrary to a noted good use as swab fluid, ethyl on the other side is presented as decontaminant and lyses catalyst ${ }^{[11]}$ which means it has a destructive effect in opposition to recovery usefulness. However such encounters that interfere with sample evidence are well interpreted during DNA profile from extraction to electrophoretic visualization. Furthermore, depending on sample state (DNA molecule size) agarose and acrylamide reaction are also studied to respond differently ${ }^{[12-14]}$ Despite substantial trials on alcohol fluid, assessment of specified ethyl (70\%) against distilled water swab for effective 
swabbing and its comparative reaction on agarose and acrylamide electrophoresis is yet established. This study, therefore, dedicates investigation on the efficient use of a $70 \%$ ethylated swab supply by using spectacle evidence convinced by reasons discussed in next section.

\section{Why 70\% Ethylated Alcohol swab?}

The percentage of alcohol on the treatment of biological sample has a direct effect relation. On DNA extraction and preservation usage, $70 \%$ ethyl has evidenced a flexible treatment of biological samples which allow morphological exploration afterward ${ }^{[15]}$. This is in contrast to high or low percentage in respect to volatile, drying blow-away, nonflexibility performance, and degradation prone. Also; compared to other alcohol groups such as isopropanol, ethanol is highly precipitous that resuspend DNA pellet easily. Catalyse insolubility of DNA molecules by forming $\mathrm{H}$-bonds with water during isolation (decrease hydration ability of water to DNA) reduced decaying. Lower dielectric lead DNA to aggregate and concentrate with cat-ions below lighter molecules under phenol-chloroform extraction ${ }^{[16,17]}$. Referring to the percentage used in the reference above, ethylated alcohol swab envisions a special use in recovering DNA trace evidence, $70 \%$ ethyl alcohol swab is optimized for consideration as explained earlier. This ground prompted on the exploration of the usefulness of $70 \%$ ethylated swab through the admission of spectacle as useful and reliable as a suggested source of trace biological evidence based on Locard's and Kick's contact and silent witness respective principles. Appreciation of $70 \%$ ethyl swab and admission of spectacle in addition to normal referred evidence such as clothes, knife, vehicles, firearms, bedding, food, condoms, lip cosmetics, wallets, jewellery, glass, skin, bullet, paper, cables, windows and door lockers/handle, stones and watch $^{[2]}$ broaden exhibits. Either in the recovery of such micro to macro exhibits like hair, dust, soil, glass particles, fluids, touched surfaces, clothes ${ }^{[1]}$ as forensic evidence, limited information is on spectacles as a potential source of biological trace evidence. Apart from compilation through literature reviews of the established useful properties of the ethyl alcohol in forensic evidence; this study complement finding used to recover trace DNA from spectacles especially through the use of $70 \%$ saturation. Aggregation of this information potentiates establishing special $70 \%$ ethyl swab for trace evidence swabbing recovery as reported in this study that compared to most recommended water swab.

\section{MATERIALS AND METHODS :}

Present Study was conducted at Human Genetic laboratory of Airlangga University to recover trace DNA from spectacles of two voluntary participants. Two sterilized cotton swabs were taken for sample collection. One swab was a readymade $70 \%$ ethylated which swabbed one spectacle and other was dry swab moistened by 1cc of distilled water and swabbed the second spectacle. Swabbing researchers used sterilized gloves and squeezed extra fluid from swabs to avoid sample loss. Swabs were immediately soaked in 2 different tubes, filled with 4cc distilled water for overnight to allow down settling of DNA biological traces. For DNA extraction uppermost fluids was removed while down settled sample solution retained. Then $0.5 \mathrm{cc}$ of each sample was isolated in a sterile centrifuge tube; pipetted with 1cc of DNAZol (Invitrogen, ThermoFisher Scientific, Waltham, MA, USA), vortexed and incubated for 15 minutes. Then vortexed with $0.2 \mathrm{cc}$ of Chloroform (Merck KGaA, 64271 Darmstadt, Germany) followed by a centrifuge of $8,000 \mathrm{rpm}$ for 10 minutes. The separated supernatant was obtained into Eppendorf with isopropanol 1cc (EMSURE®, Merck KGaA, 64271 Darmstadt, Germany) followed by 15 minutes incubation and centrifuge at $12,000 \mathrm{rpm}$ for 10 minutes then with care followed by discard of supernatant fluid again leaving settled and concentrated pellet. The pellet was washed with $0.5 \mathrm{cc}$ of $70 \%$ ethanol (EMSURE®, Merck KGaA 64271 Darmstadt, Germany) followed by 15 minutes incubation and then centrifuge at $12,000 \mathrm{rpm}$ for 5 minutes which again followed $^{[18]}{ }^{[19]}$ by removal of supernatant. Finally, 50 $\mu 1$ of distilled water resuspend formed DNA pellet for spectrophotometer and electrophoresis.
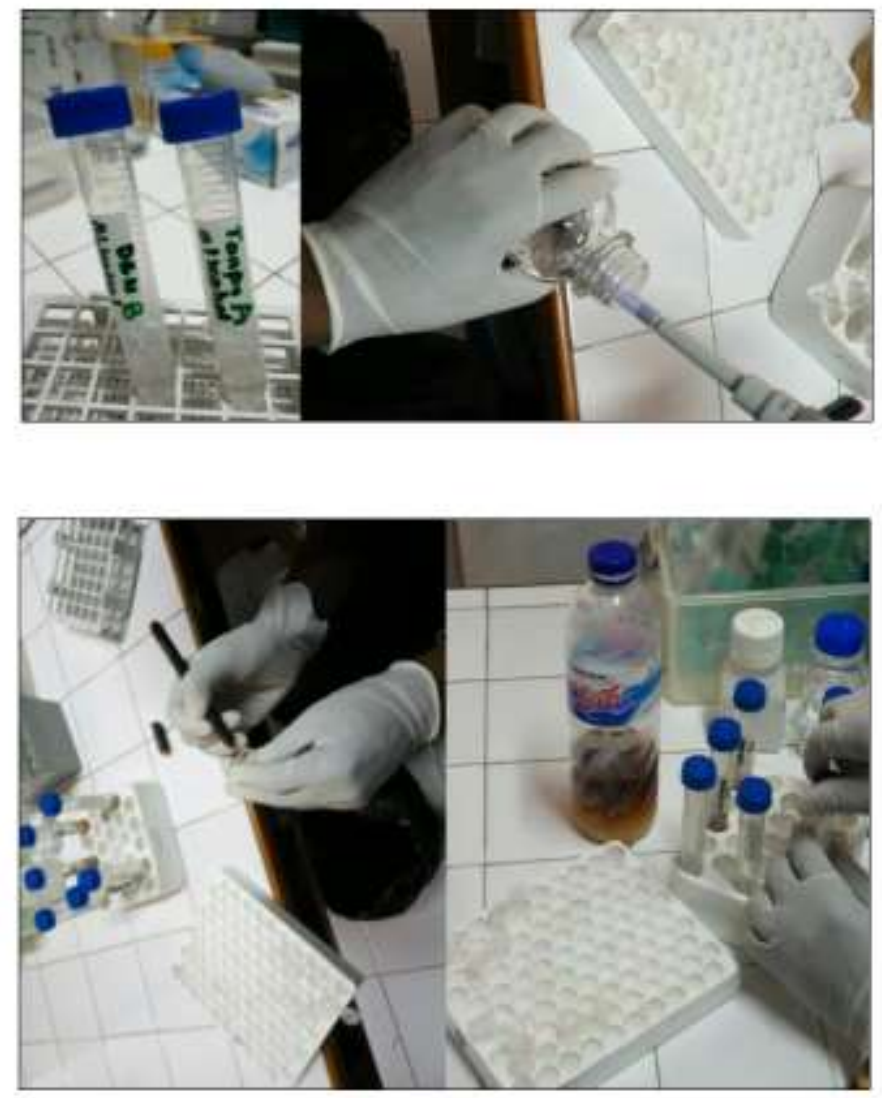

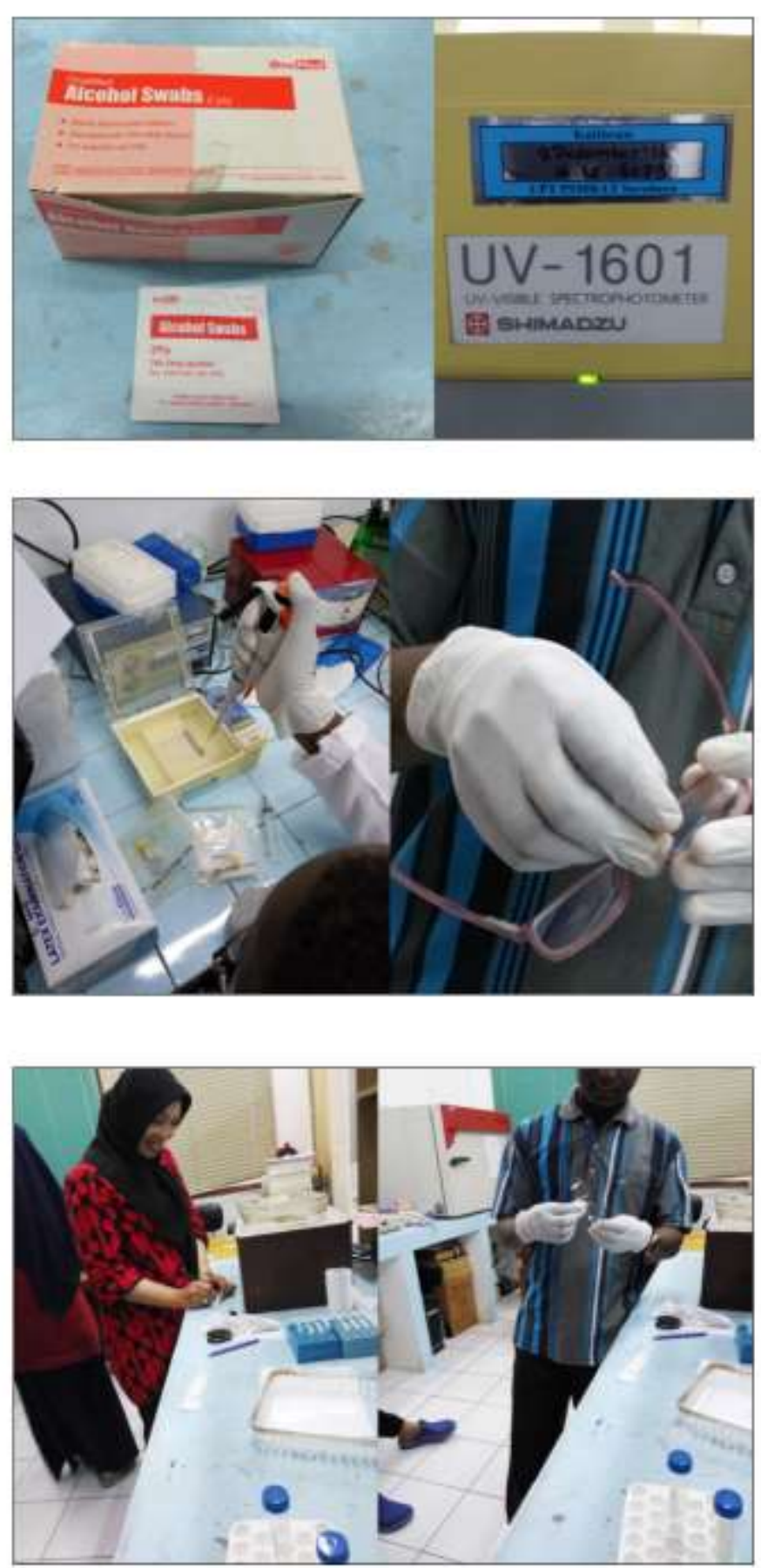

\section{Spectrophotometer Measurements}

Quantification spectrophotometry measurement read in order to establish concentration and quality parameters of $70 \%$ ethylated swab in reference to distilled water swab but also the potential extent of spectacle evidence in yielding significant biological sample for DNA. Using Ultravioletvisible Spectrophotometer (UV-1601, PC, Shimadzu, Japan) DNA Concentration was given by absorbance reading at 260nm and 280 in UV-1061 and DNA Purity was given by Optical Density (OD) OD260/OD280 ratio, refer Table 1
Table 1 : Concentration and Purity of Spectacle DNA evidence on the ethylated and non-ethylated swab

\begin{tabular}{|l|c|c|c|c|}
\hline $\begin{array}{l}\text { Sample } \\
\text { Code }\end{array}$ & $\begin{array}{l}\text { Absorbance } \\
\mathbf{2 6 0} \mathbf{~ n m}\end{array}$ & $\begin{array}{l}\text { Absorbance } \\
\mathbf{2 8 0} \mathbf{~ n m}\end{array}$ & $\begin{array}{l}\text { DNA } \\
\text { Concentration } \\
\text { (ng/ul) }\end{array}$ & $\begin{array}{l}\text { DNA } \\
\text { Purity }\end{array}$ \\
\hline $\begin{array}{l}\text { Distilled } \\
\text { Water } \\
\text { Swab-A }\end{array}$ & 0.187 & 0.159 & 654.5 & 1.176 \\
\hline $\begin{array}{l}\mathbf{7 0 \%} \\
\text { Ethylated } \\
\text { Swab-B }\end{array}$ & 0.406 & 0.333 & 1421 & 1.219 \\
\hline
\end{tabular}

\section{Electrophoresis}

Electrophoresis involved both acrylamide and agarose gel without Polymerase Chain Reaction (PCR) (purposely to find out precise and accurate of $70 \%$ ethylated swab method and spectacle referenced to distilled water swab for recovery and profile of trace $\mathrm{D} N$ A). Percentage preparation and concentration decision of these two gels is by the size of fragments measured and needed to be resolved for optimal separation for visualization. The measured DNA fragments concentration (Table 1), decided acrylamide gel to be prepared at $6 \%$ concentration then stained with silver nitrate while agarose was at $2 \%$ concentration with ethidium bromide stain then visualized in Ultraviolet transilluminator with reference to $^{[13]}$. As to the reference made, $6 \%$ acrylamide concentration is determined between $80-800 \mathrm{bp}$ of native to $50-500$ of denatured DNA while $2 \%$ agarose concentration is determined between 200-1500bp. Charged molecules then responded in accordance with visualization of Figure 3.

Figure 1 : Concertration of DNA extracted from $70 \%$ ethyl and distilled water swabbed spectacle.

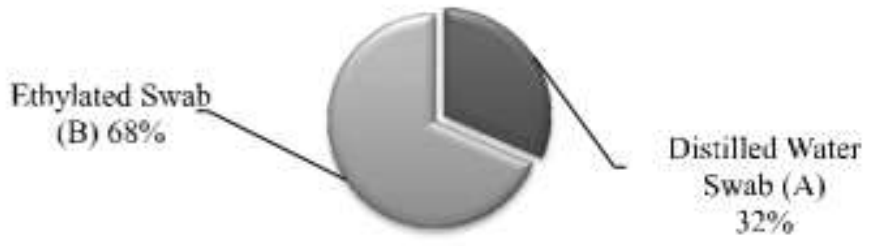

Figure 2 : Purity of DNA Extracted from 70\% Ethyl and Distilled Water Swabbed Spectacle. DNA Purity

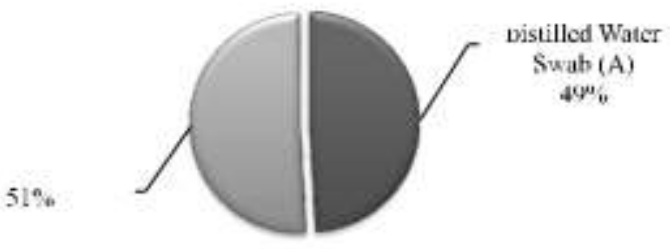


Figure 3 : Electrophoretic reaction between $6 \%$ acrylamide and $2 \%$ agarose gel from a DNA sample recovered through $\mathbf{7 0 \%}$ ethyl and distilled water swabbed spectacle evidence.

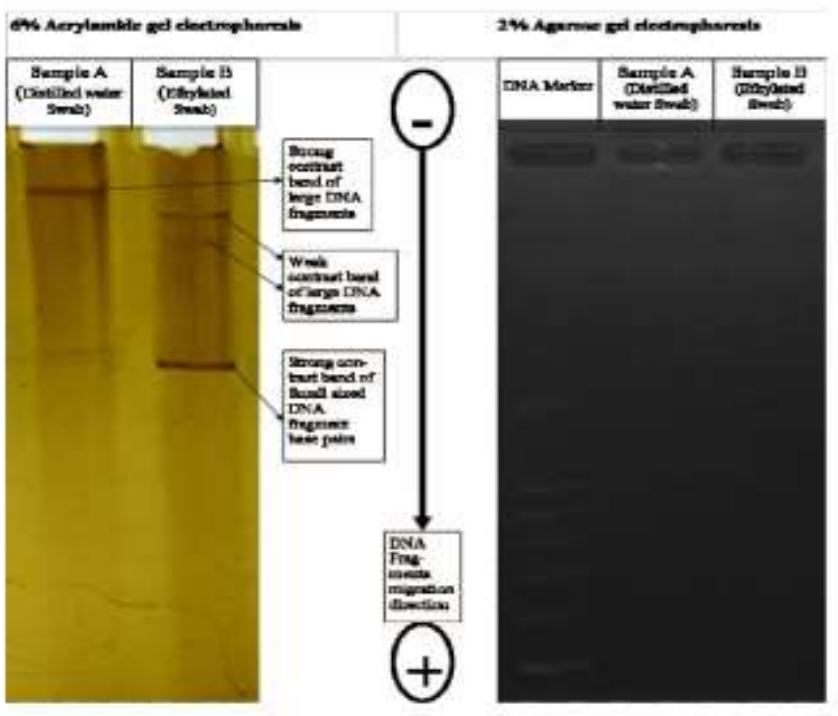

\section{Measures to control the study}

Contamination and control measures of analyzing foreign and un-targeted genetic adhered. The adhered measure included the use of sterile supply and equipment, one-sided and direction swabbing, constant laboratory temperature at 26.5 oC, use of PPE gears, use of maintained and calibrated of machines together with twice a week sterilization and disinfection of the laboratory room by Ultraviolet lamps.

\section{RESULTS AND DISCUSSION :}

\section{$\mathbf{7 0 \%}$ Ethyl swab recovery method}

According to literature, alcoholic swab has exemplified usefulness in varied percent. As from this study, a $70 \%$ is reasonably examined and presented to substitute and establish a useful ethylated swab potential to maximizing recovery of trace biological evidence. Compared the two used swab: $70 \%$ ethyl alcoholic swab and distilled water swab, finding presented a more closely results in spectrophotometer measurement (Table 1) but quite different in electrophoresis band contrast (Figure 3) between the swabs at different gel electrophoresis. Concentration reading measured almost three times in $70 \%$ ethylated swab compared to distilled water swab as per Figure 1 presentation. This concentration gives an interpretation that, $70 \%$ ethylated swab recover more DNA sample compared to possible amount able to be recovered by distilled water swab. In forensic profiling analysis, this interpretation gives a meaning to the usefulness of increased probability and assured recovering the adequate amount of sample from the targeted evidence of traces that is potential to enable successful profiling results during experimentation.
Deducing purity measurement; spectrophotometry purity increases confidence in the usage of the ethylated swab. Estimated chances of increased degradation and destruction of genetic materials as anticipated through previous few reported application in disinfection and decontamination is a contrary scenario. According to the studies; used ethyl as destructive agent forecasted expectation that this study also mighty generate a lowered and minimized purity by the fact of its destructing ability (micro-organisms discussed similar to the structure of traces). The purity of the $70 \%$ ethylated swab recovered sample is nearly similar above distilled water swab. Despite the fact that both $70 \%$ ethylated swab and distilled water swab are below the recommended limits of purity (1.6-2.0) (Table 1), the findings are promising that use of modern extraction and isolation techniques would purify to acceptable limits. The nature of results measured project useful pellet (of acceptable limit) upon with agreed contribution of effective recovery of ethyl swab. Generation of these findings proposes a step forward in utilizing the significance and modernized extraction and amplification reaction that would segregate Ribonucleic acid (RNA) and protein ingredients to attain worthy and sufficient DNA decisive profile from $70 \%$ ethylated swab.

\section{Electrophoretic reaction on $6 \%$ Acrylamide and 2\% Agarose Gel}

DNA fragments resulted from isolation and electrophoresis under both acrylamide and agarose gels without PCR are presented as seen in Figure 3. Despite the fact that PCR and sequencing technique amount to accuracy and discriminatory efficiency of genetic profile the cost consequences and technology always hinder accessibility to struggling community, this study explored the option of effective and affordable electrophoresis only techniques. Aiming to profile establishment, the study used an isolated DNA pellet from two different swabs (70\% ethylated and distilled water) into the two electrophoretic gel with the standard similar experimental condition. From the two gels type of electrophoresis, 2\% agarose electrophoretic gel didn't respond to visualization of $\mathrm{b}$ a nd s resultedfrom D N fragments migration irresponsiveness, Figure 3. The failure is established to be due to the ability to work better and resolving many large molecules compared to minute fragments, hence suitable to high sized DNA. This characteristic property is also associated with lowresolution ability - meaning to challenge reaction when amplification of fragmented, or minute molecules is skipped. These findings complement findings established and discussed by different contributors ${ }^{[12,13]}$.

Compared to acrylamide gel electrophoresis, DNA fragments mobility is observed by bands contrast stain. The success of 
$6 \%$ acrylamide gel is supported by its resolution and a higher ability to analyses of small DNA molecules. In such case, acrylamide gets preference to this traced DNA by being more sensitive to the ability to resolve small molecules. Successfulness of acrylamide gel is well appreciated on both ethylated and distilled swab, variation and responding differences are certainly by swabbing technique. This acrylamide electrophoresis of DNA band contrast appearance to both swabs sample is by fragments migration effect of polarization and sizing. Migration of DNA fragments from negatively charged electrodes to positive is established in different numerous band level especially to sample of the $70 \%$ ethyl swab as presented in Figure 3. These band contrasts portray the size, length and strength of the DNA extracted from these two kinds of swabs (ethylated and distilled water swabs). As discussed in concentration and purity above (Table 1),

DNA obtained through $70 \%$ ethylated swab is with nearly similar purity but higher amount as portrayed in Figure $1 \& a m p ; 2$, this information gives the connotation that both swabs yielded pure DNA capable to be analyzed in electrophoresis, Figure 2. The meaning is that through this electrophoresis without PCR amplification of DNA targeted locus, DNA has successfully been electrophorized as full charged fragments and migrated to appropriate contrast level. However, from the displayed bands, ethylated swab contrast more bands compared to water swab; this implies that $70 \%$ ethyl swab recover more amount of DNA sample with various strength and size leading to a differed migration of which small sized fragments are lighter and migrate faster with contrast level closer to Anode electrode refer Figure 3. The longer and larger sized DNA fragments recovered in the ethylated swab and water swab appear to contrast closer to the cathode electrode due to the slower migration of charged fragments. The findings interpret varied implication of argument; first, both swabs (70\% ethylated and distilled water) recover significant amount but ethylated swab recover more to significant minute traces evidenced by different band contrast and even concentrated more at a lower level (closer to positive end). Second, the purity of the DNA sample recovered by ethylated swab is higher with excess concentration compared to water swab. Third, minute and increased extraction of the sample are of useful quality as being able to be profiled on electrophoresis even without PCR primer amplification.

Due to this fact establishment, both $2 \%$ agarose and acrylamide gel electrophoresis are challenged by stature and nature of profiled DNA molecules due to the absence of PCR amplification. Though the two gel present with varied quality and profile response, acrylamidestand out compared to agarose electrophoresis . By this result, $6 \%$ acrylamide is recommended to work far better and remain a suggested option even in absence of PCR and amplification techniques for genetic profile and DNA discrimination among individuals.

\section{Spectacle}

As other evidence found at crime scene, through this study; spectacle is evaluated to useful and potential evidence able to be used as a source of trace DNA for profiling as result of contact from a human that used before. Through a wellestablished recovery method, spectacle exhibit can significantly contribute the logged and harbored amount of DNA in a contacted sample from specific individual used before with basic observation established from non-PCRed electrophoresis.

\section{CONCLUSION :}

DNA spectrophotometer and band visualization contrast under $6 \%$ acrylamide gel depict and suggest; $70 \%$ ethylated swab to be a useful and strong method that recover large and enough sample for DNA profile establishment. As for these reasons stated above in signifying conduction of this study, $70 \%$ of ethyl is in that manner recommended due to being a flexible percent that tolerates further morphological treatment of DNA sample from recovery to temporal storage before processing in the laboratory. The study also brings attention to successful electrophoresis in consideration of profiling DNA even in the absence of or limited PCR technique by using $6 \%$ acrylamide gel. Finally, usage of spectacle as a potential source of DNA sample either found at the crime scene for criminal linkage or to purposed forensic inquiry investigative profiling.

\section{Measures to Control the Study}

Contamination and control measures of analyzing foreign and un-targeted genetic adhered. Then adhered measure included the use of sterile supply and equipment, one-sided and direction swabbing, constant laboratory temperature at 26.5 o C, use of PPE gears, use of maintained and calibrated of machines together with twice a week sterilization and disinfection of the laboratory room by Ultraviolet lamps.

\section{Abbreviations}

DNA: Deoxyribonucleic Acid; OD: Optical Density; PCR: Polymerase Chain Reactions; RNA:

Ribonucleic Acid; TBE: Tris-borate-EDTA

\section{Acknowledgements}

Authors convey gratitude to volunteers who provided spectacles for study execution, Human Genetic laboratory staff for technical assistance and Mr. Stanley Elias for English and language improvement.

Funding : None

Conflict of Interest : No conflicting interest 


\section{REFERENCE :}

Fisher BAJ, Fisher DR, Kolowski J. Forensics demystified. McGraw-Hill; 2007. 272 p.

Ah Van Oorschot R, Ballantyne KN, Mitchell RJ. Forensic trace DNA: a review. Investig Genet. 2010;1:14.

Van Oorschot R, Phelan DG, Furlong S, Scarfo GM, Holding NL, Cummins MJ. Are you collecting all the available DNA from touched objects? Int Congr Ser. 2003;1239(C):803-7.

Jack Dillon, Debra Figarelli, David Sylvester WT. Collecting DNA Evidence at Property Crime Scenes. DNA Initiative. 2009;1-30.

Oregon State Police. Physical Evidence Manual. 2015.

Puritan. Swabbing for Trace Evidence vs. Swabbing for Blood and Other Fluids. 2016. Available from: https://blog.puritanmedproducts.com/swabbing-fortrace-evidence-vs-swabbing-for-fluids Accessed on Jan 11,2018

Adamowicz MS, Stasulli DM, Sobestanovich EM, Bille TW. Evaluation of methods to improve the extraction and recovery of DNA from cotton swabs for forensic analysis. PLoS One. 2014;9(12):1-18.

Raymond JJ, van Oorschot RAH, Walsh SJ, Roux C. Trace DNA analysis: Do you know what your neighbour is doing?. A multi-jurisdictional survey. Forensic Sci Int Genet. 2008;2(1):19-28.

Slrichantrawonq K, Rerkamnuaychoke B, Ramathibodi M. DNA retrieval on flip-flops. :30-6.

Hildebrand DP, Shamji F, Yamashita B, Wilkinson D. DNA Sampling from the Trigger and Handgrip of Discharged Firearms. 2004;1-10

Gršković B, Zrnec D, Popović M, Petek MJ, Primorac D, Mršić G. Effect of ultraviolet $\mathrm{C}$ radiation on biological samples. Croat Med J. 2013;54(3):263-71.

Taylor CY, Guillén IA, Nazabal M, Fernández JR, Silva JA. Electrophoretic techniques applied to the detection and analysis of the human microsatellite DG10s478. J Biomol Tech. 2007;18(5):298-305.

Barril P, Nates S. Introduction to Agarose and Polyacrylamide Gel Electrophoresis Matrices with Respect to Their Detection Sensitivities. In: Gel Electrophoresis - Principles and Basics. Magdeldin S(editor)

Stellwagen NC. Electrophoresis of DNA in agarose gels, polyacrylamide gels and in free solution. Electrophoresis. 2010;30(Suppl 1):1-14.
Oswald N. The Basics: How Ethanol Precipitation of DNA and RNA Works. Bitesize Bio. 2007;1-15. Available from: https://bitesizebio.com/253/the-basicshow-ethanol-precipitation-of-dna-and-rna-works. Accessed on Jan. 15, 2018

Brennan J. What Does Ethanol Do in a DNA Extraction?. S ciencing.com.2017. Availablefrom: https://sciencing.com/ethanol-do-DNA-extraction8336005.html. Accessed on Jan. 15, 2018

Zumbo P. Ethanol Precipitation. Weill Cornell Med Coll. 2013;1932(Pauling):1-12.

Chen H, Rangasamy M, Tan SY, Wang H, Siegfried BD. Evaluation of five methods for total DNA extraction from western corn rootworm beetles. PLoS One. 2010;5(8).

Chomczynski P, Mackey K, Drews R, Wilfinger W. DNAzol: A reagent for the rapid isolation of genomic DNA. Biotechniques. 1997;22(3):550-3. 
